\title{
A treatment strategy for sex offenders in Japan
}

\author{
Kumiko Ando* \\ Department of Neuropsychiatry, St. Marianna University School of Medicine, Japan
}

\section{Introduction}

Examining the crime statistics for Japan [1], we see that while the number of criminal arrests has been declining since 2003, sex offenses are on the rise. Recidivism rates are also high for sex offenders $(27.7 \%$ for rape and $32.3 \%$ for sexual battery) [2,3], so there are efforts to prevent repeat offenses in Japan. Although sex offenses are often committed by people with intellectual disabilities, nearly all rehabilitation programs are designed for individuals with no such disabilities [4], and there is insufficient treatment and support for offenders as they reenter society. We therefore developed a rehabilitation program that can also be used with sex offenders that have intellectual and mental disabilities.

\section{Characteristics of rehabilitation programs for sex offenders in Japan}

Treatment for sex offenders is centered on methods such as cognitive-behavioral therapy and relapse prevention $[5,6]$, which have been shown to be effective [7,8]. Developed in United Kingdom, Sex Offender Treatment Services Collaborative- Intellectual Disabilities : SOTSEC-ID [9], is a treatment model aimed those with intellectual disabilities who are at high risk for committing sex offenses; the effectiveness of that model has also been reported [10]. We referenced the SOTSEC-ID model while developing Sex Offender Preventive Intervention and Re-integrative Treatment Schema : SPIRiTS: [11], a support program suited to the environment and needs of Japan. SPIRiTS is based on the seven pillars indicated in Figure 1 and consists of 20 two-hour sessions. An important feature of SPIRiTS is that it can be implemented by the social workers who support local communities, not just by experts in psychology or sex-offender rehabilitation.

Specifically, the program incorporates general sex education in its early stages. That is because people with intellectual and developmental disabilities often have an insufficient or erroneous understanding of sex. This problem is exacerbated in Japan, where an extreme aversion to topics of a sexual nature prevents a correct understanding of sex from being conveyed. SPIRiTS also includes tools for evaluating a person's sexual preferences and lack of understanding, allowing the factors (needs) that can lead to sex offenses to be determined on an individual basis [12]. In order to encourage the reentry of offenders to society, it contains Mindfulness-based cognitive therapy: MBCT [13] for distracting the mind from sexual urges and replacing them with thoughtful consideration as well as elements of the Good Lives Model $[14,15]$ that match concrete rehabilitation plans.

\section{From a judicial perspective to one of general clinical psychology}

Performing a detailed assessment of an individual's criminal risks and providing tangible assistance toward their daily lives-in other words, helping them "live easier"-is a critical component of treating and supporting sex offenders. It is also useful for offenders to acquire specific skills by utilizing behavioral therapy that helps them control their actions when they are experiencing increased sexual urges.

We implemented the SPIRiTS program on 6 sex offenders. The program incorporates cognitive-behavioral therapy that separates thought (understanding), emotion, and behavior from one other to break the vicious cycle and relaxation therapy. At first, there was concern that cognitive-behavioral therapy would be difficult for people with intellectual disabilities, but the response to workbooks that contained many illustrations and role-playing exercises was favorable. Some members of the treatment program improved their personal relationships with others through the relaxation therapy and reported that they even received positive evaluations at work. In our research results, all participants scored higher on the happiness scale and exhibited improvements in self-respect.

\section{Conclusion}

When people break the law, they receive punishment. They must not only face their crimes but confront their personal weak points as well. The most important thing for overcoming those weak points, however, is the question of how to best utilize their individual strengths. All treatment is built on a foundation of trust and security. It is our challenge now to prevent repeat offenses and to reintegrate offenders into society rather than to exclude them.

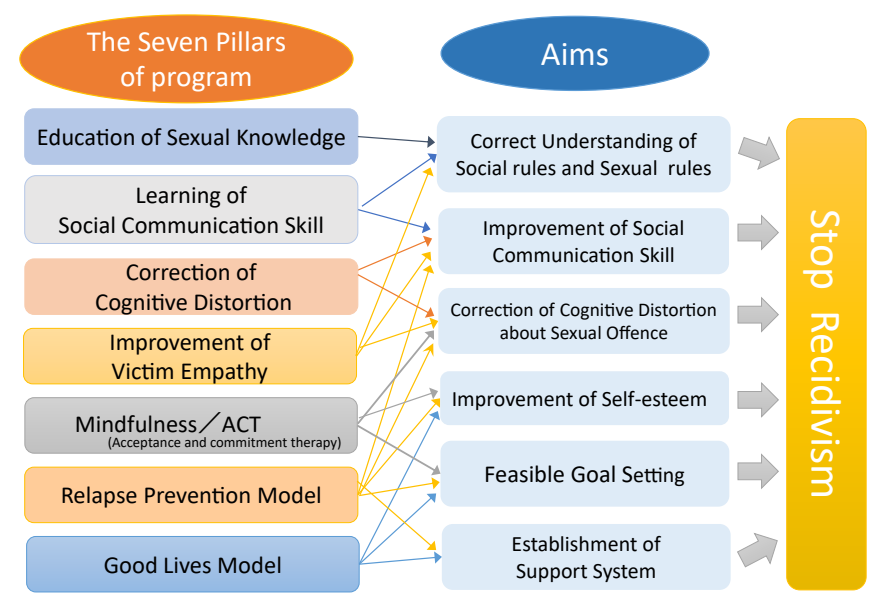

Figure 1. Seven pillars and aims on SPIRITS

${ }^{\star}$ Correspondence to: Kumiko Ando, Department of Neuropsychiatry, St. Marianna University School of Medicine, Japan, E-mail: ando@marianna-u.ac.jp

Received: May 15, 2020; Accepted: May 26, 2020; Published: May 29, 2020 


\section{References}

1. White Paper on Crime 2017 (http://hakusyo1.moj.go.jp/jp/64/nfm/mokuji.html)

2. White Paper on Crime 2015 (http://hakusyo1.moj.go.jp/jp/62/nfm/mokuji.html)

3. Fehrenbach PA, Smith W, Monastersky C, Deisher RW (1986) Adolescent sexual offenders: offender and offense characteristics. Am J Orthopsychiatry 56: 225-233. [Crossref]

4. Craig LA, Stringer I, Sanders CE (2012) Treating sexual offenders with intellectua limitations in the community. British Journal of Forensic Practice 14: 5-20

5. Ward T (2000) PartIII. Conceptual and clinical Revisions. In: Laws DR, Hudson SM, Ward T (eds), Remaking Relapse Prevention with Sex Offenders: A Sourcebook (pp77-142).

6. Lipsey MW, Landenberger NA, Wilson SJ (2007) Effects of cognitive-behavioral programs for criminal offenders. Campbell Systematic Reviews. Cited 2018-5-13.

7. Wheeler JG, George WH, Marlatt GA (2006) Relapse Prevention for Sexual Offenders: Considerations for the "Abstinence Violation Effect". Sex Abuse 18: 233-248. [Crossref]

8. Nomura K, Yamamoto T, Hayashi K (2011) Effects of Psychosocial Factors on Cognitive Behavior Therapy for Preventing Recidivism by Sex Offenders : A MetaAnalysis. Jap J Behavior Ther 37: 143-155.
9. SOTSEC-ID HP (https://www.kent.ac.uk/tizard/sotsec/)

10. Heaton KM, Murphy GH (2013) Men with intellectual disabilities who have attended sex offender treatment groups: a follow-up J Appl Res Intellect Disab 26: 489-500

11. Ando K, Terumoto M (2017) Therapeutic approach to recidivism prevention for sex offenders development of "SPIRiTS". Houritsu no Hiroba 70: 49-58.

12. Ando K (2018) Up to Date on Sex Offender Treatment in Japan: Therapeutic Intervention Approach for Sex Offenders: Development and Practice of SPIRiTS. Acto criminologiae et medicinae lagalis Japonica 84:161-170.

13. Veves A, Gotink RA, Chu P, Busschbach, Jan JV, et al. (2015) "Standardised Mindfulness-Based Interventions in Healthcare: An Overview of Systematic Reviews and Meta-Analyses of RCTs". PLOS ONE 10: 4. [Crossref]

14. Good Lives Model HP (https://www.goodlivesmodel.com/)

15. Barendregt CS, Van der Laan AM, Bongers IL, Nieuwenhuizen CV (2018) Quality of life, delinquency and psychosocial functioning of adolescents in secure residential care: testing two assumptions of the Good Lives Model. Child Adolesc Psychiatry Ment Health 12: 4. [Crossref]

Copyright: (C2020 Ando K. This is an open-access article distributed under the terms of the Creative Commons Attribution License, which permits unrestricted use, distribution, and reproduction in any medium, provided the original author and source are credited. 